\title{
Two new methods for constructing double-ended priority queues from priority queues
}

\author{
Amr Elmasry • Claus Jensen · Jyrki Katajainen
}

Received: 10 August 2007 / Accepted: 2 October 2008 / Published online: 19 November 2008 C The Author(s) 2008. This article is published with open access at Springerlink.com

\begin{abstract}
We introduce two data-structural transformations to construct doubleended priority queues from priority queues. To apply our transformations the priority queues exploited must support the extraction of an unspecified element, in addition to the standard priority-queue operations. With the first transformation we obtain a double-ended priority queue which guarantees the worst-case cost of $O(1)$ for find-min, find-max, insert, extract; and the worst-case cost of $O(\lg n)$ with at most $\lg n+O(1)$ element comparisons for delete. With the second transformation we get a meldable double-ended priority queue which guarantees the worst-case cost of $O$ (1) for find-min, find-max, insert, extract; the worst-case cost of $O(\lg n)$ with at most $\lg n+O(\lg \lg n)$ element comparisons for delete; and the worst-case cost of $O(\min \{\lg m, \lg n\})$ for meld. Here, $m$ and $n$ denote the number of elements stored in the data structures prior to the operation in question.
\end{abstract}

Keywords Data structures · Priority queues · Double-ended priority queues · Min-max priority queues · Priority deques · Meticulous analysis · Comparison complexity

Mathematics Subject Classification (2000) $\quad 68 \mathrm{P} 05 \cdot 68 \mathrm{P} 10 \cdot 68 \mathrm{~W} 40 \cdot 68 \mathrm{Q} 25$

The work of the authors was partially supported by the Danish Natural Science Research Council under contracts 21-02-0501 (project Practical data structures and algorithms) and 272-05-0272 (project Generic programming-algorithms and tools). A. Elmasry was supported by Alexander von Humboldt fellowship.

\section{A. Elmasry}

Max-Planck Institut für Informatik, Saarbrücken, Germany

C. Jensen · J. Katajainen $(\varangle)$

Datalogisk Institut, Københavns Universitet, Universitetsparken 1, 2100 Copenhagen East, Denmark e-mail:jyrki@diku.dk 


\section{Introduction}

In this paper, we study efficient realizations of data structures that can be used to maintain a collection of double-ended priority queues. The fundamental operations to be supported include find-min, find-max, insert, and delete. For single-ended priority queues only find-min or find-max is supported. A (double-ended) priority queue is called meldable if it also supports operation meld. Even though the data-structural transformations to be presented are fully general, our main focus is on the comparison complexity of double-ended priority-queue operations. Throughout this paper we use $m$ and $n$ to denote the number of elements stored in a data structure prior to an operation and $\lg n$ as a shorthand for $\log _{2}(\max \{2, n\})$. Many data structures $[2,3,5$, $7-11,17-21,23-25]$ have been proposed for the realization of a double-ended priority queue, but none of them achieve $\lg n+o(\lg n)$ element comparisons per delete, if findmin, find-max, and insert must have the worst-case cost of $O(1)$.

We use the word RAM as our model of computation as defined in [15]. We assume the availability of instructions found in contemporary computers, including built-in functions for allocating and freeing memory. We use the term cost to denote the sum of instructions, element constructions, element destructions, and element comparisons performed.

When defining a (double-ended) priority queue, we use the locator abstraction discussed in [14]. A locator is a mechanism for maintaining the association between an element and its current position in a data structure. A locator follows its element even if the element changes its position inside the data structure.

Our goal is to develop realizations of a double-ended priority queue that support the following operations:

find-min $(Q)($ find-max $(Q))$. Return a locator to an element that, of all elements in double-ended priority queue $Q$, has a minimum (maximum) value. If $Q$ is empty, return a null locator.

$\operatorname{insert}(Q, p)$. Add an element with locator $p$ to double-ended priority queue $Q$.

extract $(Q)$. Extract an unspecified element from double-ended priority queue $Q$ and return a locator to that element. If $Q$ is empty, return a null locator.

delete $(Q, p)$. Remove the element with locator $p$ from double-ended priority queue $Q$ (without destroying the element).

Of these operations, extract is non-standard, but we are confident that it is useful, for example, for data-structural transformations. The following operations may also be provided.

$\operatorname{meld}(Q, R)$. Move all elements from double-ended priority queues $Q$ and $R$ to a new double-ended priority queue $S$, destroy $Q$ and $R$, and return $S$.

decrease $(Q, p, x)$ (increase $(Q, p, x))$. Replace the element with locator $p$ by element $x$, which should not be greater (smaller) than the old element. 
Any double-ended priority queue can be used for sorting (say, a set of size $n$ ). So if find-min (find-max) and insert have a cost of $O(1)$, delete must perform at least $\lg n-O(1)$ element comparisons in the worst case in the decision-tree model. Similarly, as observed in [21], if find-min (find-max) and insert have a cost of $O(1)$, increase (decrease) must perform at least $\lg n-O(1)$ element comparisons in the worst case. Recall, however, that single-ended priority queues can support find-min, insert, and decrease (or find-max, insert, and increase) at the worst-case cost of $O(1)$ (see, for example, [6]).

\subsection{Previous approaches}

Most realizations of a (meldable) double-ended priority queue — but not all — use two priority queues, minimum priority queue $Q_{\min }$ and maximum priority queue $Q_{\max }$, that contain the minimum and maximum candidates, respectively. The approaches to guarantee that a minimum element is in $Q_{\min }$ and a maximum element in $Q_{\max }$ can be classified into three main categories [10]: dual correspondence, total correspondence, and leaf correspondence. The correspondence between two elements can be maintained implicitly, as done in many implicit or space-efficient data structures, or explicitly relying on pointers.

In the dual correspondence approach a copy of each element is kept both in $Q_{\min }$ and $Q_{\max }$, and clone pointers are maintained between the corresponding copies. Using this approach Brodal [5] showed that find-min, find-max, insert, and meld can be realized at the worst-case cost of $O(1)$, and delete at the worst-case cost of $O(\lg n)$. Asymptotically, Brodal's double-ended priority queue is optimal with respect to all operations. However, as pointed out by Cho and Sahni [9], Brodal's double-ended priority queue uses almost twice as much space as his single-ended priority queue, and the leading constant in the bound on the complexity of delete is high (according to our analysis the number of element comparisons performed in the worst case is at least $4 \lg n-O(1)$ for the priority queue and $8 \lg n-O(1)$ for the double-ended priority queue).

In the total correspondence approach, both $Q_{\min }$ and $Q_{\max }$ contain $\lfloor n / 2\rfloor$ elements and, if $n$ is odd, one element is kept outside these structures. Every element $x$ in $Q_{\text {min }}$ has a twin $y$ in $Q_{\max }$, that stored at $x$ is no greater than that stored at $y$, and there is a twin pointer from $x$ to $y$ and vice versa. Both Chong and Sahni [10] and Makris et al. [21] showed that with this approach the space efficiency of Brodal's data structure can be improved. Now the elements are stored only once so the amount of extra space used is nearly cut in half. The results reported in $[10,21]$ are rephrased in Table 1.

A third possibility is to employ the leaf correspondence approach, where only the elements stored at the leaves of the data structures used for realizing $Q_{\min }$ and $Q_{\max }$ have their corresponding twins. This approach is less general and requires that some type of tree is used to represent the two priority queues. Chong and Sahni [10] showed that Brodal's data structure could be customized to rely on the leaf correspondence as well, but the worst-case complexity of delete is still about twice as high as that in the original priority queue. 
Table 1 Complexity of general transformations from priority queues to double-ended priority queues

\begin{tabular}{|c|c|c|c|}
\hline \multirow[t]{2}{*}{ Complexity } & \multicolumn{3}{|l|}{ Reference } \\
\hline & {$[10,21]$} & This paper, Sect. 2 & This paper, Sect. 3 \\
\hline$C_{\text {find-min }}^{n}$ & $c_{\text {find-min }}^{n / 2}+O(1)$ & $2 \cdot c_{\text {find-min }}^{n}+O(1)$ & $c_{\text {find-min }}^{n / 2}+O(1)$ \\
\hline$C_{\text {insert }}^{n}$ & $2 \cdot c_{\text {insert }}^{n / 2}+O(1)$ & $\begin{array}{l}O(1) \cdot c_{\text {extract }}^{n} \\
\quad+O(1) \cdot c_{\text {insert }}^{n}+O(1)\end{array}$ & $2 \cdot c_{\text {insert }}^{n / 2}+O(1)$ \\
\hline$C_{\text {extract }}^{n}$ & Not supported & $\begin{array}{l}O(1) \cdot c_{\text {extract }}^{n} \\
\quad+O(1) \cdot c_{\text {insert }}^{n}+O(1)\end{array}$ & $2 \cdot c_{\text {extract }}^{n / 2}+2 \cdot c_{\text {decrease }}^{n / 2}+O(1)$ \\
\hline$C_{\text {delete }}^{n}$ & $\begin{array}{l}2 \cdot c_{\text {delete }}^{n / 2} \\
\quad+2 \cdot c_{\text {insert }}^{n / 2}+O(1)\end{array}$ & $\begin{array}{l}c_{\text {delete }}^{n}+O(1) \cdot c_{\text {extract }}^{n} \\
\quad+O(1) \cdot c_{\text {insert }}^{n}+O(1)\end{array}$ & $\begin{array}{l}c_{\text {delete }}^{n / 2}+c_{\text {extract }}^{n / 2}+c_{\text {insert }}^{n / 2} \\
\quad+2 \cdot c_{\text {decrease }}^{n / 2}+O(1)\end{array}$ \\
\hline$C_{\text {meld }}^{m, n}$ & $\begin{array}{l}2 \cdot c_{\text {meld }}^{\lceil m / 2\rceil, n / 2} \\
\quad+2 \cdot c_{\text {insert }}^{m / 2}+O(1)\end{array}$ & Not supported & $2 \cdot c_{\text {meld }}^{\lceil m / 2\rceil, n / 2}+2 \cdot c_{\text {insert }}^{m / 2}+O(1)$ \\
\hline Extra space & $n+O(1)$ words & $\begin{array}{l}(4 / 3) n \text { elements } \\
O(\lg n) \text { words } \\
3 n \text { bits }\end{array}$ & $\begin{array}{l}n+O(1) \text { words } \\
n \text { bits }\end{array}$ \\
\hline
\end{tabular}

Here $C_{o p}^{n}$ denotes the worst-case cost of double-ended priority-queue operation $o p$ for a given problem size $n$, and $c_{o p}^{n}$ the corresponding cost of the priority-queue operation $o p$. Throughout the paper, we assume that functions $c_{o p}^{n}$ are non-decreasing and smooth, i.e. that for non-negative integers $m$ and $n, m \leq n \leq 2 m$, $c_{o p}^{m} \leq c_{o p}^{n} \leq O(1) \cdot c_{o p}^{m}$. Naturally, if $c_{\text {find-max }}^{n}=c_{\text {find-min }}^{n}$, then $C_{\text {find-max }}^{n}=C_{\text {find-min }}^{n}$

In addition to these general transformations, several ad-hoc modifications of existing priority queues have been proposed. These modifications inherit their properties, like the operation repertoire and the space requirements, directly from the modified priority queue. Most notably, many of the double-ended priority queues proposed do not support general delete, meld, nor insert at the worst-case cost of $O(1)$. Even when such priority queues can be modified to provide insert at the worst-case cost of $O(1)$, as shown by Alstrup et al. [1], delete would perform $\Theta(\lg n)$ additional element comparisons as a result.

\subsection{Efficient priority queues}

Our data-structural transformations are general, but to obtain our best results we rely on our earlier work on efficient priority queues [12,13]. Our main goal in these two earlier papers was to reduce the number of element comparisons performed by delete without sacrificing the asymptotic bounds for the other supported operations. In this paper, we use these priority queues as building blocks to achieve the same goal for double-ended priority queues.

Both our data-structural transformations require that the priority queues used support extract, which extracts an unspecified element from the given priority queue and returns a locator to that element. This operation is used for moving elements from 
one priority queue to another and for reconstructing a priority queue incrementally. Many existing priority queues can be easily extended to support extract. When this is not immediately possible, the borrowing technique presented in $[12,13]$ may be employed.

The performance of the priority queues described in $[12,13]$ is summarized in the following lemmas.

Lemma 1 [12] There exists a priority queue that supports find-min, insert, and extract at the worst-case cost of $O(1)$; and delete at the worst-case cost of $O(\lg n)$ including at most $\lg n+O(1)$ element comparisons.

Lemma 2 [13] There exists a meldable priority queue that supports find-min, insert, extract, and decrease at the worst-case cost of $O(1)$; delete at the worst-case cost of $O(\lg n)$ including at most $\lg n+O(\lg \lg n)$ element comparisons; and meld at the worst-case cost of $O(\min \{\lg m, \lg n\})$.

\subsection{Our results}

In this paper, we present two transformations that show how priority queues can be employed to obtain double-ended priority queues. With our first transformation we obtain a data structure for which all fundamental operations are nearly optimal with respect to the number of element comparisons performed. With our second transformation we obtain a data structure that also supports meld.

In our first transformation, we divide the elements into three collections containing elements smaller than, equal to, and greater than a partitioning element. It turns out to be cheaper to maintain a single partitioning element than to maintain many twin relationships as done in the correspondence-based approaches. When developing this transformation we were inspired by the priority queue described in [22], where a related partitioning scheme is used. The way we implement partitioning allows efficient deamortization; in accordance our bounds are worst-case rather than amortized in contrast to the bounds derived in [22]. Our second transformation combines the total correspondence approach with an efficient priority queue supporting decrease. This seems to be a new application of priority queues supporting fast decrease.

The complexity bounds attained are summarized in Table 1 . The main difference between the earlier results and our results is that the leading constant in the cost of delete is reduced from two to one, provided that the priority queues used support insert, extract, and decrease at the worst-case cost of $\mathrm{O}(1)$. By constructing double-ended priority queues from the priority queues mentioned in Lemmas 1 and 2, respectively, we get the following theorems.

Theorem 1 There exists a double-ended priority queue that supports find-min, findmax, insert, and extract at the worst-case cost of $O(1)$; and delete at the worst-case cost of $O(\lg n)$ including at most $\lg n+O(1)$ element comparisons.

Theorem 2 There exists a meldable double-ended priority queue that supports findmin, find-max, insert, and extract at the worst-case cost of $O(1)$; delete at the worstcase cost of $O(\lg n)$ including at most $\lg n+O(\lg \lg n)$ element comparisons; and meld at the worst-case cost of $O(\min \{\lg m, \lg n\})$. 


\section{Pivot-based partitioning}

In this section we show how a double-ended priority queue, call it $Q$, can be constructed with the help of three priority queues $Q_{\min }, Q_{\text {mid }}$, and $Q_{\max }$. The basic idea is to maintain a special pivot element and use it to partition the elements held in $Q$ into three candidate collections: $Q_{\min }$ holding the elements smaller than pivot, $Q_{\text {mid }}$ those equal to pivot, and $Q_{\max }$ those larger than pivot. Note that, even if the priority queues are meldable, the resulting double-ended priority queue cannot provide meld efficiently.

To illustrate the general idea, let us first consider a realization that guarantees good amortized performance for all modifying operations (insert, extract, and delete). We divide the execution of the operations into phases. Each phase consists of max $\left\{1,\left\lfloor n_{0} / 2\right\rfloor\right\}$ modifying operations, if at the beginning of a phase the data structure stored $n_{0}$ elements (initially, $n_{0}=0$ ). At the end of each phase, a restructuring is done by partitioning the elements using the median element as pivot. That way we ensure that at any given time-except if there are no elements in $Q$ - the minimum (maximum) element is in $Q_{\min }\left(Q_{\max }\right)$ or, if it is empty, in $Q_{\text {mid }}$. Thus all operations of a phase can be performed correctly.

Now it is straightforward to carry out the double-ended priority-queue operations by relying on the priority-queue operations.

find-min $(Q)($ find-max $(Q))$. If $Q_{\min }\left(Q_{\max }\right)$ is non-empty, the minimum (maximum) of $Q_{\min }\left(Q_{\max }\right)$ is returned; otherwise, the minimum of $Q_{\text {mid }}$ is returned.

$\operatorname{insert}(Q, p)$. If the element with locator $p$ is smaller than, equal to, or greater than pivot, the element is inserted into $Q_{\min }, Q_{\text {mid }}$, or $Q_{\max }$, respectively.

$\operatorname{extract}(Q)$. If $Q_{\min }$ is non-empty, an element is extracted from $Q_{\min }$; otherwise, an element is extracted from $Q_{\text {mid }}$.

delete $(Q, p)$. Depending on in which component $Q_{\min }, Q_{\operatorname{mid}}$, or $Q_{\max }$ the element with locator $p$ is stored, the element is removed from that component. To implement this efficiently, we assume that each node of the priority queues is augmented by an extra field that gives the name of the component in which that node is stored.

A more detailed description of the operations on $Q$ is given in Fig. 1 .

After each modifying operation it is checked whether the end of a phase is reached, and if this is the case, a partitioning is carried out. To perform the partitioning efficiently, all the current elements are copied to a temporary array $A$. This is done by employing extract to repeatedly remove elements from $Q$. Each element is copied to $A$ and temporarily inserted into another data structure $P$ for later use. We chose to implement $P$ as a priority queue to reuse the same structure of the nodes as $Q$. A linear-time selection algorithm [4] is then used to set pivot to the value of the median element in array A. Actually, we rely on a space-efficient variant of the standard prune-and-search algorithm described in [16, Sect. 3.6]. For an input of size $n$, the extra space used by this variant is $O(\lg n)$ words. After partitioning, $A$ is destroyed, 


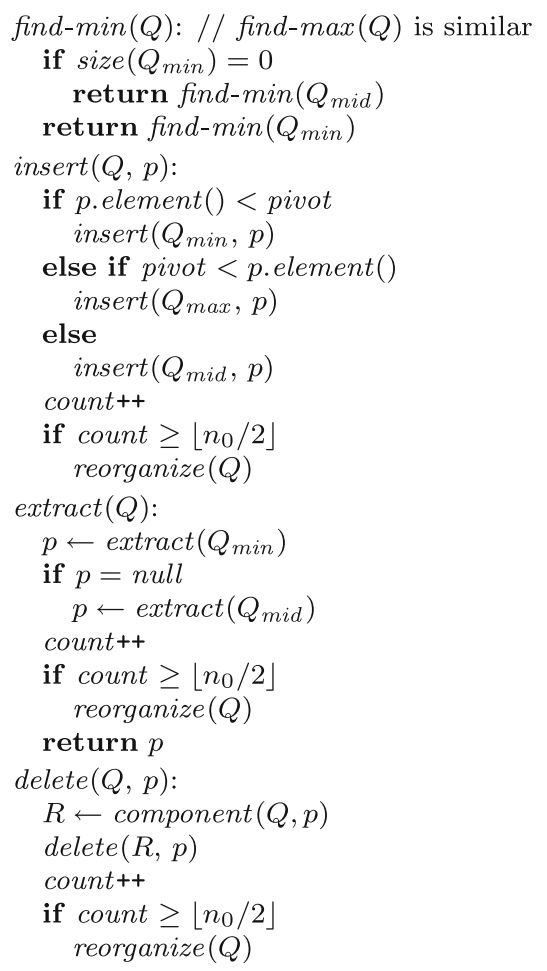

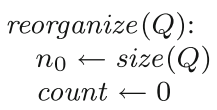

Fig. 1 This pseudo-code implements the amortized scheme. The subroutine $\operatorname{component}(Q, p)$ is assumed to return the component of $Q$ in which the element with locator $p$ is stored

and $Q$ is reconstructed by repeatedly re-extracting the elements from the temporary structure $P$ and inserting them into $Q$ (using the new pivot).

Assuming that priority-queue operations insert and extract have a cost of $O(1)$, the restructuring done at the end of a phase has the worst-case cost of $O\left(n_{0}\right)$. So a single modifying operation can be expensive, but when the reorganization work is amortized over the $\max \left\{1,\left\lfloor n_{0} / 2\right\rfloor\right\}$ operations executed in a phase, the amortized cost is only $O$ (1) per modifying operation.

Next we consider how we can get rid of the amortization. In our deamortization strategy, each phase consists of $\max \left\{1,\left\lfloor n_{0} / 4\right\rfloor\right\}$ modifying operations. We maintain the following size invariant: If at the beginning of a phase there are $n_{0}$ elements in total, the size of $Q_{\min }\left(Q_{\max }\right)$ plus the size of $Q_{\text {mid }}$ is at least $\max \left\{1,\left\lfloor n_{0} / 4\right\rfloor\right\}$. This guarantees that the minimum (maximum) element is in $Q_{\min }\left(Q_{\max }\right)$ or, if it is empty, in $Q_{\text {mid }}$. Throughout each phase, three subphases are performed in sequence; each subphase consists of about equally many modifying operations.

In the first subphase, the $n_{0}$ elements in $Q$ at the beginning of the phase are to be incrementally copied to $A$. To facilitate this, we employ a supplementary data structure $P$ that has the same form as $Q$ and is composed of three components $P_{\text {min }}, P_{\text {mid }}$, and $P_{\text {max }}$. Accompanying each modifying operation, an adequate number of elements is copied from $Q$ to $A$. This is accomplished by extracting an element from $Q$, copying 
the element to $A$, and inserting the node into $P$ using the same pivot as in $Q$. An insert directly adds the given element to $P$ without copying it to $A$, and a delete copies the deleted element to $A$ only if that element is in $Q$. At the end of this subphase, $A$ stores copies of all the elements that were in $Q$ at the beginning of the phase, and all the elements that should be in the double-ended priority queue are now in $P$ leaving $Q$ empty.

In the second subphase the median of the elements of $A$ is found. That is, the selection is based on the contents of $Q$ at the beginning of the phase. In this subphase the modifying operations are executed normally, except that they are performed on $P$, while they incrementally take part in the selection process. Each modifying operation takes its own share of the work such that the whole selection process is finished before reaching the end of this subphase.

The third subphase is reserved for clean-up. Each modifying operation carries out its share of the work such that the whole clean-up process is finished before the end of the phase. First, $A$ is destroyed by gradually destructing the elements copied and freeing the space after that. Second, all elements held in $P$ are moved to $Q$. When moving the elements, extract and insert are used, and the median found in the second subphase is used as the partitioning element.

As to find-min, the current minimum can be found from one of the priority queues $Q_{\text {min }}\left(Q_{\text {mid }}\right.$ if $Q_{\min }$ is empty) or $P_{\text {min }}$ ( $P_{\text {mid }}$ if $P_{\text {min }}$ is empty). Hence, find-min (similarly for find-max) can still be carried out efficiently.

Even if the median found is exact for $A$, it is only an approximate median for the whole collection at the end of a phase. Since after freezing the contents of $A$ at most $\max \left\{1,\left\lfloor n_{0} / 4\right\rfloor\right\}$ elements are added to or removed from the data structure, it is easy to verify that the size invariant holds for the next phase.

Let us now analyse the space requirements of the deamortized construction. Let $n$ denote the present size of the double-ended priority queue, and $n_{0}$ the size of $A$. The worst case is when all the operations performed during the phase are deletions, and hence $n$ may be equal to $3 n_{0} / 4$. That is, the array can never have more than $4 n / 3$ elements. In addition to array $A$, the selection routine requires a work area of $O(\lg n)$ words and each node has to store 3 bits to record the component $\left(Q_{\min }, Q_{\text {mid }}, Q_{\text {max }}\right.$, $P_{\text {min }}, P_{\text {mid }}$, or $P_{\text {max }}$ ) the node is in.

For the deamortized scheme, it is straightforward to derive the bounds given in Table 1. By combining the results derived for the transformation (Table 1) with the bounds known for the priority queues (Lemma 1), the bounds of Theorem 1 are obtained.

\section{Total correspondence}

In this section we describe how an efficient meldable priority queue supporting extract and decrease can be utilized in the realization of a meldable double-ended priority queue $Q$. We use $Q_{\min }$ to denote the minimum priority queue of $Q$ (supporting findmin and decrease) and $Q_{\max }$ the maximum priority queue of $Q$ (supporting find-max and increase). We rely on the total correspondence approach, where each of $Q_{\min }$ and $Q_{\max }$ contains $\lfloor n / 2\rfloor$ elements, with the possibility of having one element outside 
these structures. To perform delete efficiently, instead of using two priority-queue delete operations as in $[10,21]$, we use only one delete and employ extract that may be followed by decrease and increase.

Each element stored in $Q_{\min }$ has a twin in $Q_{\max }$. To maintain the twin relationships and to access twins fast, we assume that each node of the priority queues allows an attachment of one more pointer, a twin pointer. The element that has no twin is called a singleton. Using these concepts, the double-ended priority-queue operations on $Q$ can be performed as follows (a more detailed description of the operations is given in Fig. 2):

find-min $(Q)($ find-max $(Q))$. If $Q_{\min }\left(Q_{\max }\right)$ is empty or the $\operatorname{singleton}$ of $Q$ is smaller (greater) than the minimum (maximum) element of $Q_{\min }\left(Q_{\max }\right)$, the singleton is returned; otherwise, the minimum (maximum) of $Q_{\min }\left(Q_{\max }\right)$ is returned.

insert $(Q, p)$. If $Q$ has no singleton, the element with locator $p$ is made the singleton of $Q$ and nothing else is done. If $Q$ has a singleton and the given element is smaller than the singleton, the element is inserted into $Q_{\min }$ and the singleton is inserted into $Q_{\max }$; otherwise, the element is inserted into $Q_{\max }$ and the singleton is inserted into $Q_{\min }$. Finally, the element and the singleton are made twins of each other.

$\operatorname{extract}(Q)$. If $Q$ has a singleton, it is extracted and nothing else is done. If $Q$ has no singleton, an element is extracted from $Q_{\min }$. If $Q_{\min }$ was non-empty, an element is also extracted from $Q_{\max }$. The element extracted from $Q_{\max }$ is made the new singleton, and the element extracted from $Q_{\min }$ is returned. Before this the twins of the two extracted elements are made twins of each other and their positions are swapped if necessary, and the order in $Q_{\min }$ and $Q_{\max }$ is restored by decreasing and increasing the swapped elements (if any).

$\operatorname{delete}(Q, p)$. If the element to be deleted is the singleton of $Q$, the singleton is removed and nothing else is done. If $Q$ has a singleton, the element with locator $p$ is removed from its component, the singleton is inserted into that component, and the singleton and the twin of the removed element are made twins of each other. As in extract the two twins are swapped and the order in $Q_{\min }$ and $Q_{\max }$ is restored if necessary. On the other hand, if $Q$ has no singleton, the element with locator $p$ is removed, another element is extracted from the component of its twin, the extracted element is made the new singleton, and, if necessary, the twin of the extracted element and the twin of the removed element are swapped and the order in $Q_{\min }$ and $Q_{\max }$ is restored as above.

meld $(Q, R)$. Let $S$ denote the outcome of this operation. Without loss of generality, assume that the size of $Q$ is smaller than or equal to that of $R$. If $Q$ and $R$ together have exactly one singleton, this element becomes the singleton of $S$. If they have two singletons, these are compared, the non-greater is inserted into $Q_{\min }$, the non-smaller is inserted into $Q_{\max }$, and the inserted elements are made twins of each other. After these preparations, $Q_{\min }$ and $R_{\min }$ are melded to become $S_{\min }$, and $Q_{\max }$ and $R_{\max }$ are melded to become $S_{\max }$. 


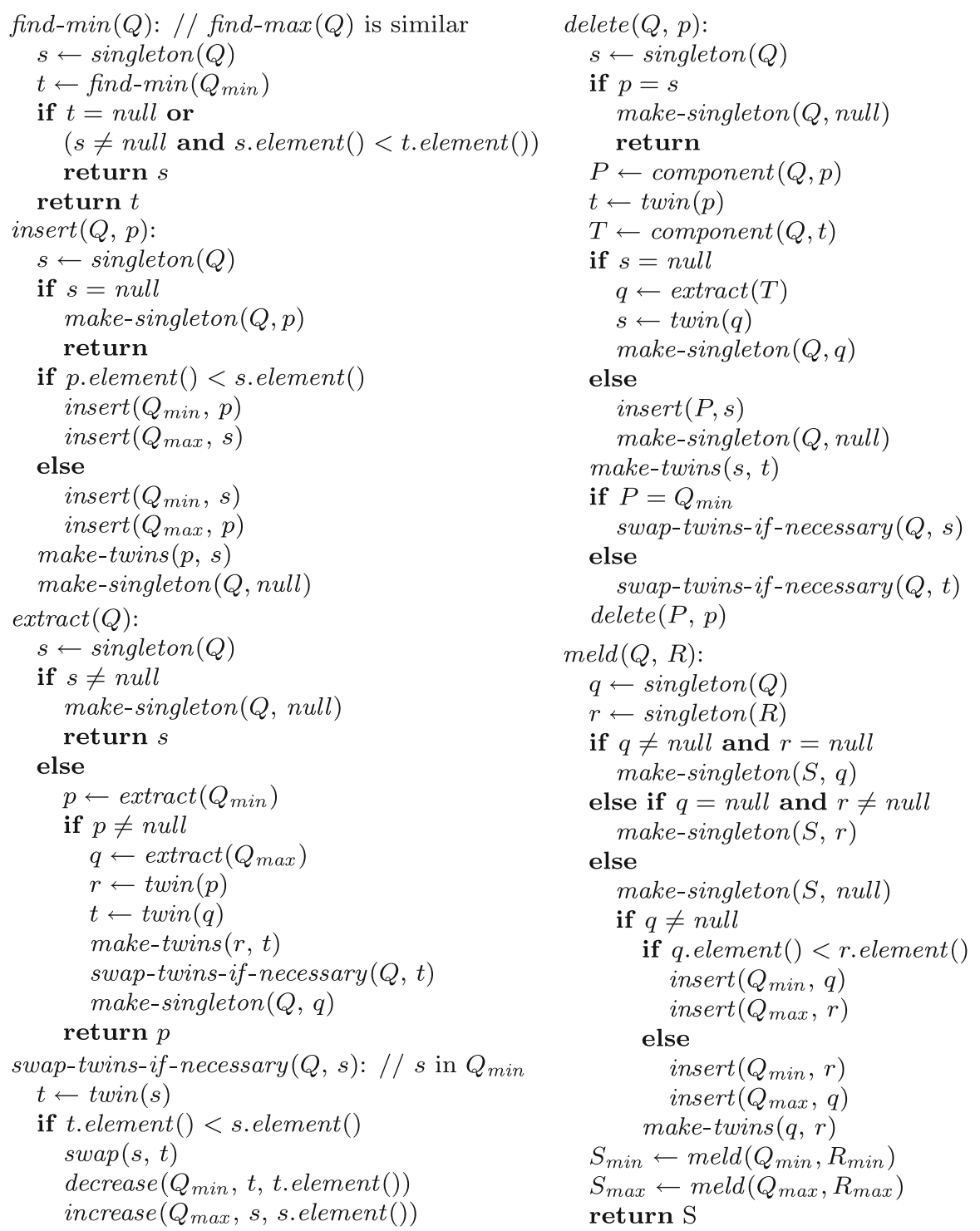

Fig. 2 This pseudo-code implements our second data-structural transformation. The subroutines used are assumed to have the following effects: $\operatorname{twin}(p)$ returns a locator to the twin of the element with locator $p ;$ make-twins $(p, q)$ assigns the twin pointers between the elements with locators $p$ and $q ; \operatorname{singleton}(Q)$ returns a locator to the singleton of $Q ;$ make-singleton $(Q, p)$ makes the element with locator $p$ the singleton of $Q$ and sets the twin pointer of $p$ to $\operatorname{null} ; \operatorname{swap}(p, q)$ puts the element with locator $p$ in place of the element with locator $q$, and vice versa; and component $(Q, p)$ returns the component of $Q$, in which the element with locator $p$ is stored. One way of implementing component is to attach to each node of $Q$ a bit indicating whether that node is in $Q_{\min }$ or $Q_{\max }$, and let insert update these bits

It is straightforward to verify that the above implementation achieves the bounds of Table 1. By combining the results of Table 1 with the bounds known for the priority queues (Lemma 2), the bounds of Theorem 2 are obtained. 


\section{Conclusions}

We conclude the paper with four open problems, the solution of which would improve the results presented in this paper.

1. One drawback of our first transformation is the extra space used for elements, and the extra element constructions and destructions performed when copying elements. The reason for copying elements instead of pointers is that some elements may be deleted during the selection process. It would be interesting to know whether the selection problem could be solved at linear cost when the input is allowed to be modified during the computation.

2. Our realization of a double-ended priority queue using the priority queues introduced in [12] works on a pointer machine, but the meldable version using the priority queues introduced in [13] relies on the capabilities of a RAM. This is in contrast with Brodal's data structure [5] which works on a pointer machine. Therefore, it is natural to ask whether random access could be avoided.

3. To obtain meld having the worst-case cost of $O(1)$, the price paid by Brodal [6] is a more expensive delete. It is unknown whether meld could be implemented at the worst-case cost of $O(1)$ such that at most $\lg n+o(\lg n)$ element comparisons are performed per delete.

4. If for a meldable double-ended priority queue meld is allowed to have the worstcase cost of $O(\min \{\lg m, \lg n\})$, it is still relevant to ask whether delete can be accomplished at logarithmic cost with at most $\lg n+O(1)$ element comparisons.

Open Access This article is distributed under the terms of the Creative Commons Attribution Noncommercial License which permits any noncommercial use, distribution, and reproduction in any medium, provided the original author(s) and source are credited.

\section{References}

1. Alstrup S, Husfeld T, Rauhe T, Thorup M (2005) Black box for constant-time insertion in priority queues. ACM Trans Algorithms 1:102-106

2. Arvind A, Pandu Rangan C (1999) Symmetric min-max heap: a simpler data structure for double-ended priority queue. Inf Process Lett 69:197-199

3. Atkinson MD, Sack J-R, Santoro N, Strothotte T (1986) Min-max heaps and generalized priority queues. Commun ACM 29:996-1000

4. Blum M, Floyd RW, Pratt V, Rivest RL, Tarjan RE (1973) Time bounds for selection. J Comput Syst Sci 7:448-461

5. Brodal GS (1995) Fast meldable priority queues. In: Proceedings of the 4th international workshop on algorithms and data structures. Lecture notes in computer science, vol 955. Springer, Berlin, pp 282-290

6. Brodal GS (1996) Worst-case efficient priority queues. In: Proceedings of the 7th ACM-SIAM symposium on discrete algorithms. ACM/SIAM, New York/Philadelphia, pp 52-58

7. Carlsson S (1987) The deap —a double-ended heap to implement double-ended priority queues. Inf Process Lett 26:33-36

8. Chang SC, Du MW (1993) Diamond deque: a simple data structure for priority deques. Inf Process Lett 46:231-237

9. Cho S, Sahni S (1999) Mergeable double-ended priority queues. Int J Found Comput Sci 10:1-18

10. Chong K-R, Sahni S (2000) Correspondence-based data structures for double-ended priority queues. ACM J Exp Algorithmics 5: article

11. Ding Y, Weiss MA (1993) The relaxed min-max heap: a mergeable double-ended priority queue. Acta Inform 30:215-231 
12. Elmasry A, Jensen C, Katajainen J (2008) Multipartite priority queues. ACM Trans Algorithms (to appear)

13. Elmasry A, Jensen C, Katajainen J (2008) Two-tier relaxed heaps. Acta Inform 45:193-210

14. Goodrich MT, Tamassia R (2002) Algorithm design: foundations, analysis, and internet examples. Wiley, Hoboken

15. Hagerup T (1998) Sorting and searching on the word RAM. In: Proceedings of the 15th annual symposium on theoretical aspects of computer science. Lecture notes in computer science, vol 1373. Springer, Berlin, pp 366-398

16. Horowitz E, Sahni S, Rajasekaran S (1998) Computer algorithms/C++. Computer Science Press, New York

17. Høyer P (1995) A general technique for implementation of efficient priority queues. In: Proceedings of the 3rd Israel symposium on the theory of computing and systems, IEEE, Los Alamitos, pp 57-66

18. Katajainen J, Vitale F (2003) Navigation piles with applications to sorting, priority queues, and priority deques. Nordic J Comput 10:238-262

19. Khoong CM, Leong HW (1993) Double-ended binomial queues. In: Proceedings of the 4th international symposium on algorithms and computation. Lecture notes in computer science, vol 762. Springer, Berlin, pp 128-137

20. van Leeuwen J, Wood D (1993) Interval heaps. Comput J 36:209-216

21. Makris C, Tsakalidis A, Tsichlas K (2003) Reflected min-max heaps. Inf Process Lett 86:209-214

22. Mortensen CW, Pettie S (2005) The complexity of implicit and space-efficient priority queues. In: Proceedings of the 9 th workshop on algorithms and data structures. Lecture notes in computer science, vol 3608. Springer, Berlin, pp 49-60

23. Nath SK, Chowdhury RA, Kaykobad M (2000) Min-max fine heaps. arXiv.org e-Print archive article cs.DS/0007043. http://arxiv.org

24. Olariu S, Overstreet CM, Wen Z (1991) A mergeable double-ended priority queue. Comput J 34: 423-427

25. Rahman MZ, Chowdhury RA, Kaykobad M (2003) Improvements in double ended priority queues. Int J Comput Math 80:1121-1129 